International Journal of Biomedicine I June 2019 - Volume 9, Issue Suppl_1: Abstracts From the Second Russian International Conference "Cryo-electron microscopy 2019: achievements and prospects"

POSTER ABSTRACT PRESENTATIONS

SESSION TITLE: STRUCTURE AND FUNCTIONS OF THE TRANSCRIPTION AND TRANSLATION APPARATUS OF THE CELL

DOI: 10.21103/IJBM.9.Suppl_1.P21

\title{
Abstract P-21: The Oligomeric State of Base Excision DNA Repair Proteins and Their Complexes Explored by Dynamic Light Scattering
}

\author{
Inna A. Vasil'eva, Nina A. Moor, Rashid O. Anarbaev, Olga I. Lavrik \\ Institute of Chemical Biology and Fundamental Medicine, Siberian Branch of the Russian Academy of \\ Sciences, Novosibirsk, Russia
}

Background: Base excision DNA repair (BER) is very efficient process that involves the action of many enzymes and accessory proteins to correct the most common DNA lesions usually induced by ionizing irradiation and oxidative stress. Coordination of BER depends on assembling protein participants into multicomponent dynamic "repairosome". Knowledge of the oligomeric states of protein complexes is useful for their structural studies. The primary aim of our work was to estimate the oligomeric states of enzymes and coordinating proteins of BER and of their complexes characterized previously by using fluorescence-based approach.

Methods: We employed dynamic light scattering (DLS) technique to measure the hydrodynamic sizes of several enzymes and proteins, DNA polymerase $\beta(\mathrm{Pol} \beta)$, apurinic/apyrimidinic endonuclease 1 (APE1), tyrosyl-DNA phosphodiesterase 1 (TDP1), X-ray repair cross-complementing protein 1 (XRCC1) and poly(ADP-ribose) polymerase 1 (PARP1), present alone or in the equimolar mixtures with each other. DLS measurements were performed in solution under true equilibrium conditions by using a Zetasizer Nano ZS instrument. Glutaraldehyde cross-linking was used to analyze the prevailing complexes in the proteins mixtures.

Results: The hydrodynamic radii of the protein particles determined from the volume weighted size distribution profiles have been used to estimate the oligomeric states of proteins and their complexes. All the proteins of different molecular weights and functions in BER have been proposed to form homodimers upon their self-association. The most probable oligomerization state of the binary complexes formed by PARP1 with various proteins is a heterotetramer (dimer of heterodimers). On the contrary, the oligomerization state of XRCC1 hetero-associated complexes has been shown to vary from the heterodimer (complex with APE1) to the heterotetramer (complexes with Pol $\beta$, TDP1 and PARP1). PARP1 was revealed to destabilize strong interaction between Pol $\beta$ and XRCC1, suggesting modulation of the proteins association mode upon assembling higher order complexes. 
Conclusion: Our study demonstrates for the first time applicability of the DLS technique for determination of the oligomeric states of various proteins and their complexes, when complemented with fluorescence-based and chemical cross-linking approaches.

Key Words: Base excision DNA repair complexes $\bullet$ dynamic light scattering $\bullet$ oligomeric state Sources of Funding: This work was supported by the RSF project 19-14-00107.

International Journal of Biomedicine. 2019;9 Suppl 1: S26. doi: 10.21103/IJBM.9.Suppl_1.P21 (C)2019 International Medical Research and Development Corporation 